\title{
Lebensstandardsicherung durch betriebliche Altersvorsorge? Deutschland im Vergleich
}

\author{
Tobias Wiß
}

\section{Einleitung}

Das 2018 in Kraft getretene Betriebsrentenstärkungsgesetz sowie die Vereinbarung der Großen Koalition Ende 2019 zur Einführung einer Grundrente haben den Diskussionen um die Ausgestaltung und künftige Entwicklung des deutschen Alterssicherungssystems Aufschwung gegeben. Überlegungen, innerhalb des staatlichen Systems eine Art nicht bedürftigkeitsgeprüfte Mindestrente einzuführen, weisen bereits darauf hin, dass es die gesetzliche Rentenversicherung (GRV) alleine nicht (mehr) schafft, vielen Personen selbst nach mehr als 30 Jahren mit Beitragszahlungen eine Rente oberhalb der Grundsicherungsschwelle zu gewährleisten, geschweige denn den Lebensstandard im Alter zu sichern. ${ }^{1}$ Diese Entwicklung kommt allerdings keineswegs überraschend, schließlich war das erklärte politische Ziel der Rentenreformen in den 2000er Jahren das Leistungsniveau der gesetzlichen Rentenversicherung zu reduzieren und gleichzeitig die Rolle der betrieblichen und privaten Altersvorsorge zu stärken.

Der internationale Vergleich zeigt, dass innerhalb eines Mehrsäulenmodells das staatliche Rentensystem die Funktion der Verhinderung von Altersarmut übernimmt, während die Lebensstandardsicherung entweder über eine staatliche Rentenversicherung und/oder über die betriebliche Altersvorsorge organisiert werden kann. Der vorliegende Beitrag analysiert die aktuelle Rolle der betrieblichen Altersvorsorge (bAV) in Deutschland und beschäftigt sich daher mit der Lebensstandardsicherung und nicht mit der Vermeidung von Altersarmut, da Letzteres in der Regel nicht in den Aufgabenbereich der bAV fällt.

Im unmittelbaren Anschluss an die Rentenreform des Jahres 2001 hat die bAV eine starke Dynamik erfahren, was Ausdruck fand in einer steigenden Zahl an bAV-Verträgen und dem Abschluss von zahlreichen Tarifverträgen insbesondere zu Details der Entgeltumwandlung. Diese Dyna-

1 Zur Privatisierung der Altersvorsorge und steigender Altersarmut vgl. Ebbinghaus 2018. 
mik ergibt sich aus der Logik, dass ein künftig geringeres Rentenniveau kompensiert werden muss, wobei der bAV neben der Riester-Rente eine zentrale Rolle eingeräumt wurde (Blank 2015). Allerdings hat diese anfängliche Dynamik inzwischen ihren Zenit überschritten und wir haben es seit mehreren Jahren mit einer Stagnation der bAV zu tun. Die ursprünglichen Erwartungen in Bezug auf die Zahl der Abschlüsse und die Höhe von Beiträgen wurden bisher nicht erfüllt. Dies hängt auch mit dem Irrglauben zusammen, dass ein auf Freiwilligkeit beruhendes System der betrieblichen und privaten Altersvorsorge einen ehemaligen Teil des verpflichtenden GRV-Systems kompensieren kann. ${ }^{2}$

Neben der Analyse der aktuellen Rolle der bAV möchte das vorliegende Kapitel auch einen Beitrag zur aktuellen Reformdiskussion und möglichen künftigen Entwicklungen leisten. Hierzu erfolgt ein Blick auf andere europäische Länder. Es wird dargelegt, wie in anderen Ländern die Lebensstandardsicherung gewährleistet wird und welche Rolle die bAV hierbei im gesamten Alterssicherungssystem spielt.

Der folgende Abschnitt 2 beschäftigt sich zunächst mit dem Zusammenspiel von staatlicher Alterssicherung und betrieblicher Altersversorgung in Deutschland und der Einbettung der bAV in das gesamte Alterssicherungssystem. Im Anschluss an jüngste Entwicklungen der GRV (Abschnitt 3) analysiert der vierte Abschnitt die Entwicklung der bAV seit 2001, wobei der Schwerpunkt auf der aktuellen Situation entlang einiger Dimensionen wie Branchenunterschieden liegt. Abschnitt 5 versucht, den deutschen Weg im Vergleich mit anderen Ländern einzuordnen und mögliche Reformoptionen abzustecken.

\section{Das Zusammenspiel von staatlicher und betrieblicher Altersversorgung}

Zur analytischen Erfassung des gesamten Alterssicherungssystems und zur Ermöglichung von Vergleichen ist es hilfreich zu unterscheiden, welche Institutionen und Akteure für welchen Teil der Alterssicherung verantwortlich sind, und welche Funktionen diese Teile für das individuelle Einkommen im Alter haben. Die Forschung zu Alterssicherungssystemen orientiert sich hierbei an einem Modell aus verschiedenen Säulen und Schichten (Ebbinghaus 2011; Immergut/Anderson 2007).

2 Zum Problem der freiwilligen Vorsorge siehe Meyer 2015; zur gescheiterten Riester-Rente vgl. Nullmeier 2015. 
Die erste Säule umfasst den staatlichen Teil des Alterssicherungssystems, in Deutschland also die GRV (siehe Tabelle 1). In der zweiten Säule wird die bAV entweder von beiden Sozialpartnern (Gewerkschaften und Arbeitgebern) oder vom Arbeitgeber alleine, gegebenenfalls unter Einbezug der betrieblichen Interessenvertretung, organisiert. In der dritten Säule schließlich finden wir Rentenprodukte wie zum Beispiel private Rentensparpläne oder Lebensversicherungen, die von einzelnen Personen direkt mit Finanzdienstleistern abgeschlossen werden. Das Konzept der ,Schicht ${ }^{6}$ bezieht sich auf die jeweilige Funktion eines Teils der gesamten Alterssicherung für das Einkommen im Alter. In der ersten Schicht geht es um die Vermeidung von Armut, was in Deutschland über die bedarfsgeprüfte Grundsicherung im Alter erfolgt. In der zweiten Schicht finden wir diejenigen Teile des Alterssicherungssystems, die für die Lebensstandardsicherung zuständig sind, also dafür sorgen, dass der erreichte Lebensstandard während der Phase der Erwerbstätigkeit auch im Alter gehalten werden kann. Hierfür ist hauptsächlich (noch) die gesetzliche Rentenversicherung zuständig. Aufgrund von Leistungskürzungen kann sie dies aber nicht mehr alleine erfüllen, daher wird zusätzlich eine betriebliche oder auch private Altersvorsorge benötigt. Die privaten Riester- und Rürup-Produkte können sowohl Teil der zweiten als auch der dritten Schicht sein. In Letzterer geht es um eine Absicherung über den Lebensstandard hinaus. Das vorliegende Kapitel behandelt im Folgenden den grau schattierten Bereich der zweiten Schicht in der zweiten Säule, also die bAV. Prinzipiell muss hier zwischen dem öffentlichen und privaten Sektor unterschieden werden. Angestellte im öffentlichen Dienst sind verpflichtend branchenweit abgesichert, wohingegen das System der bAV im privaten Sektor auf Freiwilligkeit beruht. Beamte werden im Folgenden nicht weiter berücksichtigt, da diese über eine privilegierte steuerfinanzierte Beamtenversorgung verfügen, welche die erste und zweite Schicht innerhalb der ersten Säule umfasst.

Das Zusammenspiel von staatlicher und nicht-staatlicher Alterssicherung ist durch die beiden Mechanismen des crowding out und crowding in gekennzeichnet. Dies bedeutet, dass hohe staatliche Renten betriebliche und private Renten überflüssig machen und diese daher verdrängen (crowding out). Wenn die staatlichen Renten dagegen niedrig sind oder gekürzt werden, dann gibt es einen höheren Bedarf an zusätzlicher Altersvorsorge (crowding in). Deutschland war bis Ende der 1990er Jahre stärker durch den crowding out-Mechanismus gekennzeichnet, Leistungskürzungen in der GRV haben jedoch zu einem stärkeren, politisch gewollten und geförderten „Eindringen“ der zusätzlichen Altersvorsorge in das Gesamtsystem der Alterssicherung geführt. 
Tabelle 1: Säulen und Schichten im deutschen Alterssicherungssystem

\begin{tabular}{|c|c|c|c|}
\hline & $\begin{array}{c}\text { 1. Säule } \\
\text { staatliche Altersvorsorge }\end{array}$ & $\begin{array}{l}\text { 2. Säule } \\
\text { betriebliche Altersvorsorge }\end{array}$ & $\begin{array}{c}\text { 3. Säule } \\
\text { individuelle Altersvorsorge }\end{array}$ \\
\hline & & Sozialpartner Arbeitgeber & \\
\hline $\begin{array}{l}\text { 3. Schicht } \\
\text { (top-up) }\end{array}$ & & & $\begin{array}{l}\text { private Rentenpläne, } \\
\text { Lebensversicherungen }\end{array}$ \\
\hline $\begin{array}{l}\text { 2. Schicht } \\
\text { (einkommensbezogene } \\
\text { Lebensstandardsicherung) }\end{array}$ & $\begin{array}{l}\text { gesetzliche } \\
\text { Rentenversicherung }\end{array}$ & $\begin{array}{l}\text { öffentlicher Dienst: } \\
\text { - verpflichtende VBL } \\
\text { Privatwirtschaft: } \\
\text { - fünf freiwillige } \\
\text { Durchführungswege } \\
\text { - Tarifverträge } \\
\text { - Kollektive } \\
\text { Versorgungswerke }\end{array}$ & $\begin{array}{l}\text { Riester-Rente, } \\
\text { Rürup-Rente }\end{array}$ \\
\hline
\end{tabular}

\begin{tabular}{ll}
\hline 1. Schicht & bedarfsgeprüfte \\
(Armutsvermeidung) & Grundsicherung im Alter
\end{tabular}

Quelle: Eigene Darstellung nach Ebbinghaus et al. (2011) und Schulze/Jochem (2007).

\section{Hintergrund: Die Entwicklung der gesetzlichen Rente}

Wenn es darum geht, die Leistungshöhe der GRV abzuschätzen, wird in deutschen Debatten sehr häufig auf das Netto-Sicherungsniveau vor Steuern, umgangssprachlich auch Rentenniveau genannt, verwiesen. Gemeint ist hiermit die staatliche Rente, die ein/e Versicherte/r bei 45 Versicherungsjahren mit Durchschnittsentgelt im Verhältnis zum aktuellen durchschnittlichen Arbeitnehmerentgelt erhält. Der Bruttorentenbetrag und der Durchschnittsverdienst werden hierbei um die jeweiligen Sozialversicherungsabgaben sowie der Durchschnittsverdienst zusätzlich um den durchschnittlichen Aufwand für die zusätzliche Altersvorsorge reduziert, die Steuerbelastung wird dagegen nicht berücksichtigt. Aus Abbildung 1 lässt sich ablesen, dass das Rentenniveau im Jahr 2019 48,2 \% beträgt und seit 1990 um fast 7 Prozentpunkte gesunken ist. Für das Jahr 2033 wird aktuell ein Wert von nur noch 44,6\% vorausberechnet. Das sinkende Rentenniveau geht dabei mit einem steigenden Beitragssatz einher. Der Beitragssatz liegt 2019 zwar bei 18,6 \%, also in etwa dem Wert des Jahres 1990 (18,7\%), zwischenzeitlich aber erfolgte ein Anstieg auf 20,3 \% (1997). Für 2033 geht der aktuelle Rentenversicherungsbericht von einem wesentlich höheren Beitrag (22,0\%) aus. 


\section{Abbildung 1: Entwicklung des Rentenniveaus (\%) und des Beitragssatzes (\%)}

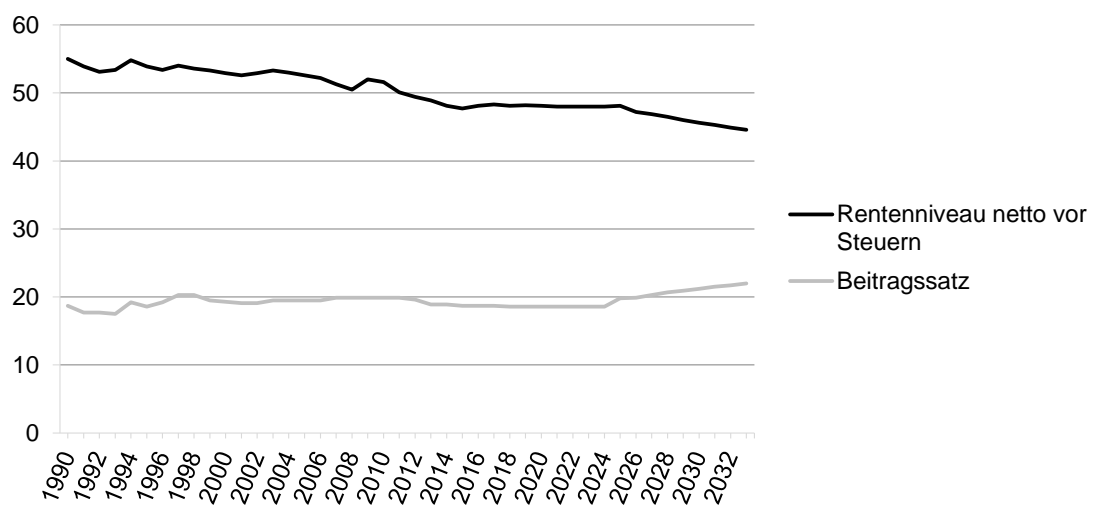

Quellen: Eigene Darstellung nach Bundesministerium für Arbeit und Soziales (2019a) und Deutsche Rentenversicherung Bund (2018).

Es muss jedoch beachtet werden, dass das Sicherungsniveau vor Steuern keine Aussagen über die Höhe des individuellen Rentenniveaus zulässt. Es handelt sich beim Netto-Sicherungsniveau vor Steuern um eine modellhafte Berechnung einer fiktiven Rentnerin bzw. eines fiktiven Rentners basierend auf 45 Entgeltpunkten, die dann nicht dem individuellen Einkommen vor Rente, sondern dem aktuellen Durchschnittsverdienst aller ArbeitnehmerInnen gegenübergestellt wird. Das bedeutet, dass das Rentenniveau ohne Veränderungen im Bereich der gesetzlichen Rentenversicherung sowohl sinken als auch steigen kann, also nicht zwangsläufig mit höheren oder niedrigeren individuellen Renten einhergeht. Dies ist beispielsweise der Fall, wenn es zu Änderungen bei den Sozialversicherungsbeiträgen und dem durchschnittlichen Arbeitnehmerentgelt kommt. Vor diesem Hintergrund kann hinterfragt werden, ob die aktuellen Haltelinien ${ }^{3}$ des Rentenversicherungs-Leistungsverbesserungs- und Stabilisierungsgesetzes aus dem Jahr 2018 auf einem geeigneten Messkonzept beruhen, wenn Veränderungen des Rentenniveaus sich nicht zwangsläufig auch auf individuelle Rentenhöhen auswirken.

3 Unter anderem darf die untere Haltelinie zwischen 2019 und $202548 \%$ und bis $203043 \%$ nicht unterschreiten. 


\section{Abbildung 2: Nettoersatzraten aus dem öffentlichen System für Deutschland (\%) bei halbem, einfachem und anderthalbfachem Duchschnitts- verdienst}

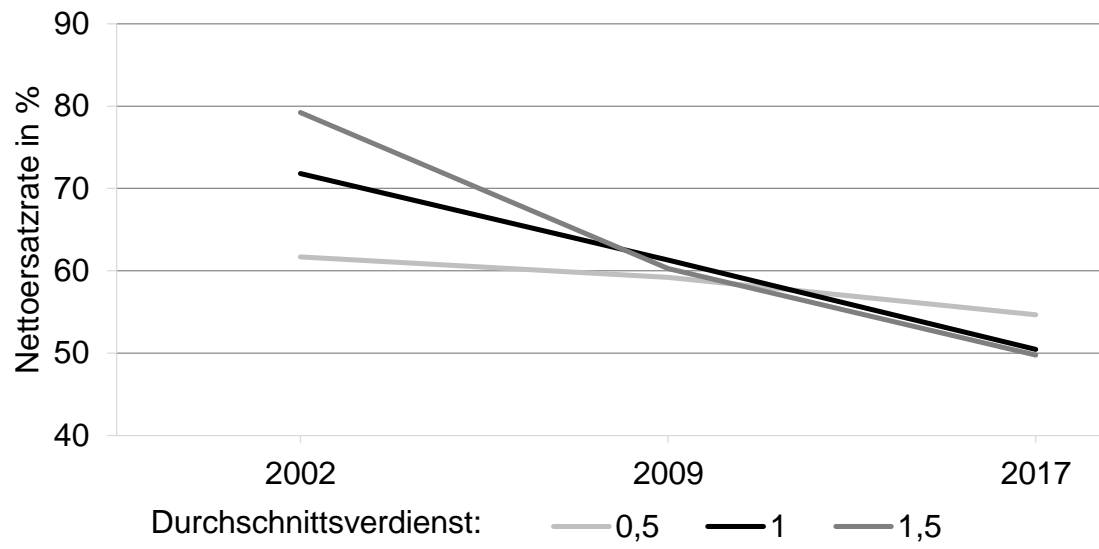

Quellen: OECD (2005, 2009, 2017).

Anmerkungen: Zwischen 2013 und 2015 kam es zu leichten Veränderungen der Modellannahmen.

Demgegenüber bezieht sich die sogenannte Ersatzrate auf das Verhältnis von individueller Rentenleistung zu individuellem Einkommen vor Renteneintritt und misst damit explizit individuelle Rentenhöhen. Dies ist insbesondere für die Messung der Lebensstandardsicherung von Bedeutung. Im Hinblick auf den Vergleich Deutschlands mit anderen Ländern in Abschnitt 5 wird hier Bezug genommen zur Kalkulation der OECD. ${ }^{4}$ Abbildung 2 zeigt die Entwicklung von simulierten Nettoersatzraten, definiert als individuelle Rentenleistung im Verhältnis zum letzten individuellen Einkommen, nach Abzug von Steuern und Sozialversicherungsbeiträgen. Der Eintritt ins Erwerbsleben erfolgt im jeweiligen Jahr im Alter von 20 Jahren und der Renteneintritt zum jeweiligen gesetzlichen Renteneintrittsalter, bzw. zum abschlagsfreien Renteneintritt für Deutschland. Auch hier wird der Rückgang seit 2002 deutlich. Kam ein Durchschnittsverdiener im Jahr 2002 noch auf eine Nettoersatzrate von 71,8\% und konnte damit nach gängiger Definition den Lebensstandard im Alter halten, so reduzier-

4 Auch die Simulationen von Bridgen/Meyer (2014) verdeutlichen das abgesunkene Rentenniveau seit 2001. 
te sich dieser Wert auf 61,3\% im Jahr 2009 und nur noch 50,5\% im Jahr 2017 (OECD 2005, 2009, 2017). Die ursprünglich über dem Durchschnitt liegende Ersatzrate für Personen mit dem 1,5fachen des Durchschnittsverdienstes (dunkelgraue Linie) ging bis 2017 wesentlich stärker zurück $(-29,4 \%)$ als die Ersatzrate von Personen mit dem 0,5fachen des Durchschnittsverdienstes (hellgraue Linie; -7\%). Die Unterschiede zwischen den Jahren können größtenteils mit Rentenreformen erklärt werden.

Wenn nun im Alter die GRV nur noch $51 \%$ des vorherigen Gehalts ersetzt, dann kann alleine mit staatlichen Leistungen der Lebensstandard nicht mehr gewährleistet werden, wobei die OECD auf das durchschnittliche Einkommen über die gesamte Erwerbskarriere abstellt und keine Veränderungen der Einkommensposition berücksichtigt. Diese Entwicklung zeigt recht deutlich, welche Lücke die Rentenreformen seit Beginn der 2000er Jahren im staatlichen Teil des Alterssicherungssystems produziert haben. Eine zusätzliche Altersvorsorge ist somit für die Lebensstandardsicherung im Alter notwendig.

\section{Die Entwicklung der betrieblichen Altersvorsorge}

Welche Entwicklung hat nun die bAV seit den Reformen Anfang der 2000er Jahre genommen und wie sieht die aktuelle Situation aus? Im Folgenden wird auf Daten und Veröffentlichungen im Rahmen der Trägerbefragung zur betrieblichen Altersversorgung im Auftrag des Bundesministeriums für Arbeit und Soziales (BMAS) sowie die Arbeitskostenerhebung des Statistischen Bundesamtes zurückgegriffen.

\subsection{Beschäftigte mit bAV}

In Abbildung 3 ist die Entwicklung der Anzahl von bAV-Anwartschaften als Anteil an allen sozialversicherungspflichtigen Beschäftigten in der Privatwirtschaft und im öffentlichen Dienst abgebildet. Jährliche Daten sind nur für Mehrfachanwartschaften verfügbar, die für das vorliegende Kapitel wichtigeren Zahlen zum Anteil von Beschäftigten mit bAV sind zum Teil lückenhaft. Der Trend scheint aber sehr ähnlich zu sein. Im Anschluss an die Rentenreform von 2001 und der Einführung des Rechtsanspruchs auf Entgeltumwandlung inklusive Steuer- und Sozialabgabenfreiheit der bAV- 
Beiträge bis zu $4 \%$ der Beitragsbemessungsgrenze kam es, wie von den meisten politischen Akteuren gewollt, zu einem starken Anstieg der bAVAnwartschaften von 52,1 \% im Jahr 2001 auf 69,4 \% im Jahr 2005.

Bei Betrachtung des gesamten Zeitraums 2001 bis 2017 (dem letzten Jahr mit verfügbaren Daten) erhöhte sich die absolute Anzahl an Beschäftigten mit bAV um 33,1\%, der Anteil der Beschäftigten mit bAV an allen sozialversicherungspflichtigen Beschäftigten dagegen nur um 14,2\%. Dies hängt mit einem stärkeren Anstieg der sozialversicherungspflichtigen Beschäftigten gegenüber den bAV-Anwartschaften zusammen. Für die Ermittlung der Abdeckung von Beschäftigten mit einer bAV-Anwartschaft ist deren Anteil an allen sozialversicherungspflichtigen Beschäftigten der aussagekräftigere Wert. Man könnte durchaus erwarten, dass die bAV-Anwartschaften mit dem Anstieg von sozialversicherungspflichtigen Beschäftigten mithalten sollte, wenn der künftige Lebensstandard im Alter nur noch in Kombination von gesetzlicher Rente und bAV erreicht werden kann. Dies ist offensichtlich nicht der Fall. Im Gegenteil, seit 2005 stagniert der Anteil der Beschäftigten mit einer bAV und ist in jüngster Zeit sogar rückläufig. Bei Herausrechnung von Mehrfachanwartschaften ist der Anteil von Beschäftigten mit einer bAV von 58,9\% im Jahr 2013 auf 55,6\% im Jahr 2017 gesunken. Dies ist von weitreichender sozialpolitischer Bedeutung. ${ }^{5}$ Nur noch etwas weniger mehr als die Hälfte aller sozialversicherungspflichtig Beschäftigten kann den Lebensstandard im Alter halten, wenn allein die bAV berücksichtigt wird. Die eigentliche Zahl ist wahrscheinlich niedriger, da wir a) keine Informationen über die Höher der Beiträge haben, also nicht wissen, ob ausreichend Beiträge eingezahlt werden und $b$ ) die Zahl der Beschäftigten mit bAV durch die Studien im Auftrag des Bundesministeriums für Arbeit und Soziales (BMAS) laut Statistischem Bundesamt leicht überhöht sind (Statistisches Bundesamt 2015a). Die im Jahr 2001 in sie gesetzten Erwartungen konnte die bAV (und im Übrigen auch die Riester-Rente) in Deutschland somit nicht erfüllen und bleibt hinter den Erwartungen zurück (Wiß 2011). Da dies jedoch nur den Durchschnitt für alle Beschäftigten darstellt, nimmt der folgende Abschnitt eine Differenzierung der Abdeckung und Beitragshöhe vor.

5 Bei den Riester-Verträgen ist in jüngster Zeit sogar ein leichter Rückgang der absoluten Zahlen zu verzeichnen; s. BMAS 2019c. 


\section{Abbildung 3: Entwicklung von bAV-Anwartschaften (in \% der sozialversiche- rungspflichtigen Beschäftigten $)^{6}$}

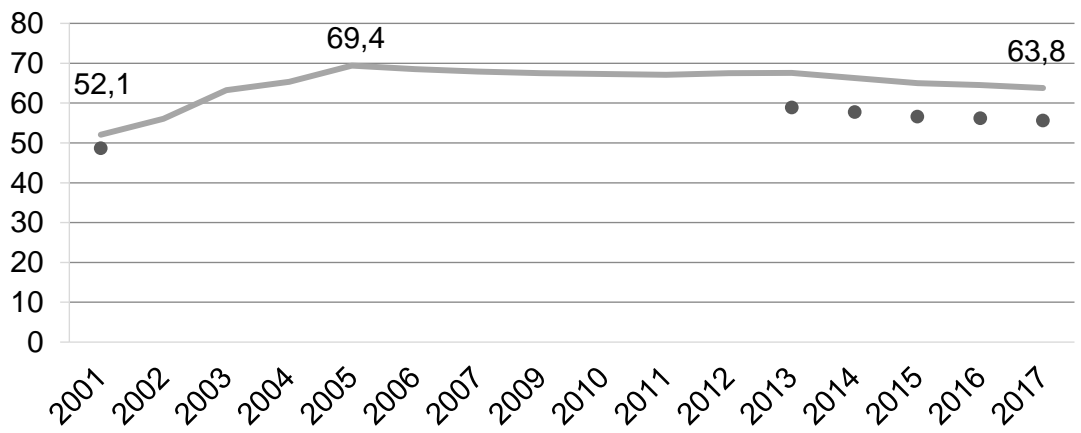

inkl. Mehrfachanwartschaften

- ohne Mehrfachanwartschaften

Quellen: Eigene Darstellung nach Bundesagentur für Arbeit (2019) und Bundesministerium für Arbeit und Soziales (2019b).

\subsection{Beiträge}

Selbst wenn Beschäftigte über eine bAV-Anwartschaft verfügen, bedeutet dies nicht automatisch, dass diese im Zusammenspiel mit der staatlichen Rente den Lebensstandard im Alter sichert. Ein großer Unsicherheitsfaktor ist die Höhe der Beiträge sowie die Entwicklung der Finanzprodukte, in die die Beiträge investiert werden. ${ }^{7}$ Der aktuelle Bericht der BMAS-Erhebung (Bundesministerium für Arbeit und Soziales 2019b) weist einen durchschnittlichen monatlichen Betrag von ca. $€ 90-€ 110$ für die Durchführungswege Pensionskassen, Pensionsfonds und Direktversicherungen aus. ${ }^{8}$ Dieser Betrag ist recht konstant über die letzten Jahre hinweg. Das heißt, es erfolgte keine signifikante Anpassung an Preis- und Lohnerhöhungen, was real mit einem Rückgang des Betrages einhergeht. Entsprechend den

6 Trotz der modellhaften Herausrechnung von Mehrfachanwartschaften können die Zahlen ohne Mehrfachanwartschaften die Gesamtzahl von Beschäftigten mit bAV überschätzen (Statistisches Bundesamt 2015a).

7 Die Höhe der Verwaltungskosten, die bei privaten Vorsorgeprodukten in der Regel höher sind als bei der gesetzlichen Rentenversicherung, sind ein weiterer Faktor bei der Ermittlung der späteren Leistung aus der bAV.

8 Für Direktzusagen und Unterstützungskassen konnten keine Beiträge ermittelt werden. 
Werten für das Jahr 2017 wird somit bei Vorliegen einer bAV durchschnittlich nur mit 1,4\%-1,7\% der Beitragsbemessungsgrenze (West) für die bAV vorgesorgt, was deutlich unter der maximalen sozialbeitragsfreien Fördersumme von $4 \%$ liegt. $^{9}$ Mit letzterem Wert, also $4 \%$ Beitragshöhe, wird zudem kalkuliert, damit die Beschäftigten, zusammen mit der GRV, den Lebensstandard im Alter halten können. Knapp doppelt so hoch sind dagegen die Beiträge für Beschäftigte im öffentlichen Dienst. Zudem steigen diese auch kontinuierlich an, was damit zusammenhängt, dass Arbeitgeber- und Arbeitnehmerbeiträge hier anteilig zum Verdienst und nicht als absoluter Betrag, wie dies in der Regel in der Privatwirtschaft festgelegt ist, in die bAV eingezahlt werden. Vorläufig kann damit festgehalten werden, dass a) nur unwesentlich mehr als die Hälfte aller sozialversicherungspflichtig Beschäftigten über eine bAV verfügen und b) diese (bzw. deren Arbeitgeber) nur knapp die Hälfte des für die Lebensstandardsicherung erforderlichen Beitrages in eine bAV-Anwartschaft einzahlen.

Im Gegensatz zu den Erhebungen im Auftrag des BMAS erlauben die Daten der Arbeitskostenerhebung des Statistischen Bundesamtes genauere Analysen, vor allem auch für die Höhe der Beiträge. Bei der Interpretation der Daten und der Abbildungen muss berücksichtigt werden, dass die Ergebnisse der BMAS-Erhebung von den Ergebnissen des Statistischen Bundesamtes abweichen.

Die Zahlen des Statistischen Bundesamtes, basierend auf einer Befragung von Unternehmen, unterschätzen die Anzahl der Beschäftigten mit einer bAV gegenüber den Zahlen des BMAS, da nur Unternehmen mit mehr als zehn Beschäftigten berücksichtigt werden. Die Untersuchungen im Auftrag des BMAS basieren zudem auf einer Befragung der bAV-Träger sowie Statistiken des Pensions-Sicherungs-Vereins auf Gegenseitigkeit (PSVaG), der Bundesanstalt für Finanzdienstleistungsaufsicht (BaFin) und des Gesamtverbandes der Deutschen Versicherungswirtschaft (GDV), wobei aufgrund von Mehrfachanwartschaften die Zahl der Beschäftigten mit bAV leicht überschätzt wird. Daraus ergibt sich, dass die durchschnittliche Höhe von Aufwendungen sowohl der Arbeitgeber als auch der ArbeitnehmerInnen durch das Statistische Bundesamt eher überschätzt wird, da die Höhe der bAV-Aufwendungen mit der Unternehmensgröße ansteigen. Zudem berücksichtigen die Zahlen des Statistischen Bundesamtes auch Direktzusagen und Unterstützungskassen - wobei die Beiträge hier in der Regel wesentlich höher als in den anderen Durchführungswegen sind -, zu denen keine Beitragshöhen in den BMAS-Berichten vorliegen. Trotz dieser

9 Zur Förderstruktur siehe BMAS 2020. 
Schwächen in Bezug auf die Gesamtanzahl von Anwartschaften und Beschäftigten mit bAV erlauben die Daten des Statistischen Bundesamtes Unterschiede zwischen West- und Ostdeutschland, Branchen und Unternehmensgrößen darzustellen.

Abbildung 4: Arbeitgeber- und Arbeitnehmeraufwendungen für die bAV 2016 je Vollzeiteinheit (\% Bruttoverdienstsumme aller Unternehmen) in West- und Ostdeutschland ${ }^{10}$

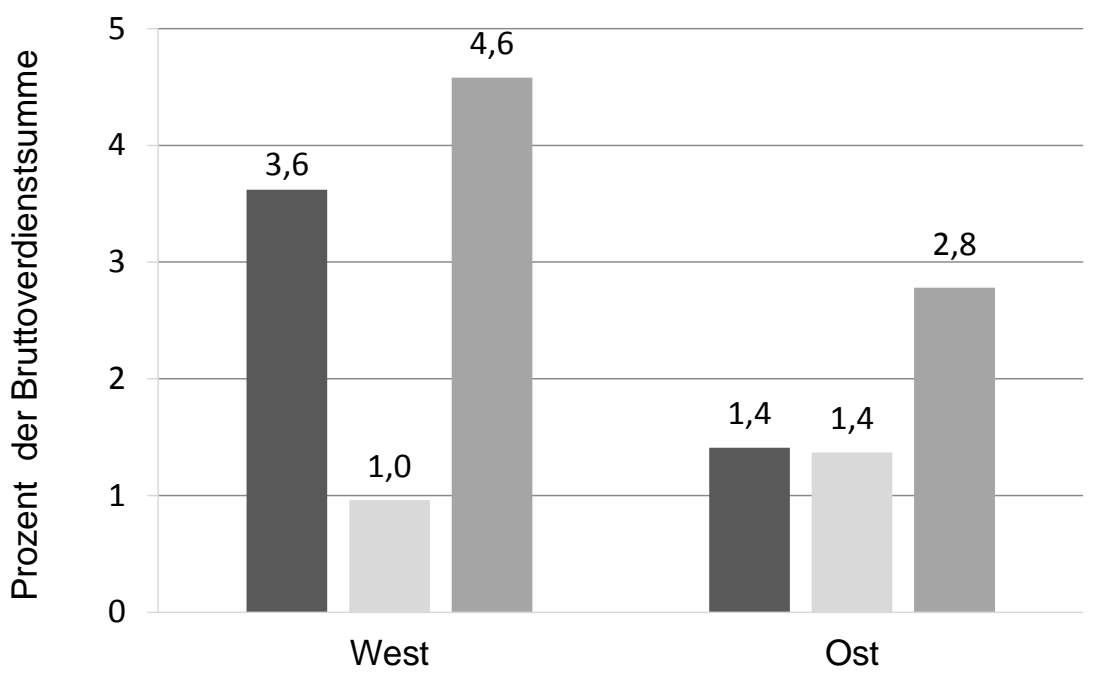

\section{$\because A G \quad A N \quad A G+A N$}

Quellen: Statistisches Bundesamt (2018).

10 AG: Arbeitgeber, AN: ArbeitnehmerInnen. Unternehmen mit mehr als 10 Beschäftigten. Arbeitgeberaufwendungen (ohne Entgeltumwandlung) umfassen arbeitgeberfinanzierte Aufwendungen für betriebliche Ruhegeldzusagen, Zuwendungen an Pensions- und Unterstützungskassen (auch Zusatzversorgungskassen sowie Zusatzversorgungseinrichtungen des öffentlichen Dienstes), Beiträge zu Direktversicherungen, Beiträge an Pensionsfonds sowie Beiträge an den Pensions-Sicherungs-Verein. Aufwendungen der Beschäftigten durch Entgeltumwandlung, Eigenbeiträge, Eigenbeteiligungen und Arbeitnehmerzulagen in allen Durchführungswegen der bAV. 
Abbildung 4 zeigt die bAV-Aufwendungen von Arbeitgebern und ArbeitnehmerInnen je Vollzeiteinheit ${ }^{11}$ für das Jahr 2016. Anteilig für die Bruttoverdienstsumme aller Unternehmen und Beschäftigten (getrennt berechnet für West- und Ostdeutschland) wandten Arbeitgeber in Westdeutschland 3,6\% und ArbeitnehmerInnen 0,96\% für die bAV auf. Der Gesamtaufwand in Westdeutschland ist wesentlich höher als in Ostdeutschland, was vor allem auf die geringeren Arbeitgeberbeiträge in Ostdeutschland zurückgeführt werden kann. Die ArbeitnehmerInnen hingegen wenden in Ostdeutschland mehr für die bAV auf (1,37\%) als ArbeitnehmerInnen in Westdeutschland (0,96\%). Die Ursache hierfür kann nicht abschließend geklärt werden, die Vermutung, dass die ArbeitnehmerInnen in Ostdeutschland eher fehlende bzw. geringe Arbeitgeberbeiträge ausgleichen, liegt jedoch nahe.

\section{Abbildung 5: Anteil von privatwirtschaftlichen Unternehmen mit bAV nach Fi- nanzierungsform (\%), Anteil an Unternehmen mit bAV}

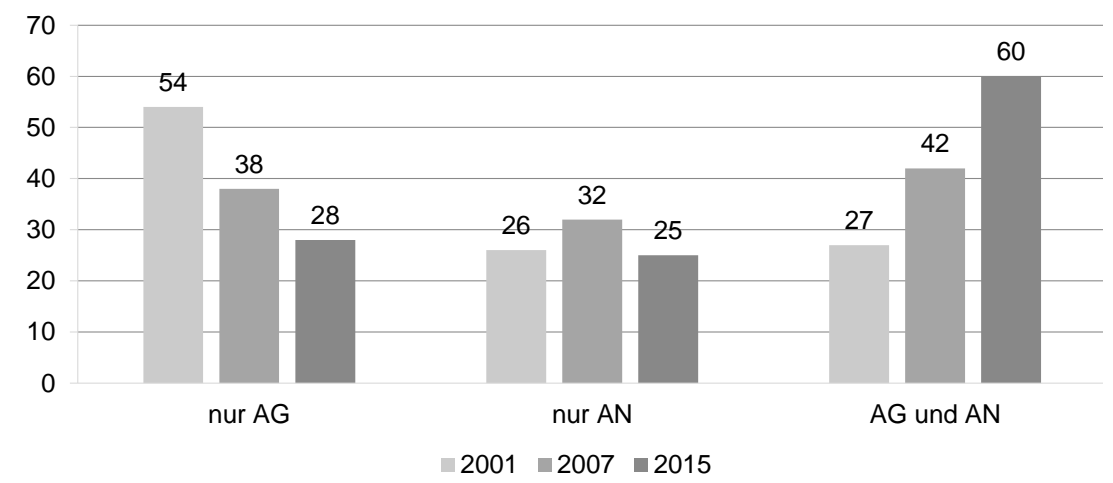

Quellen: Bundesministerium für Arbeit und Soziales (2008, 2016).

Die Daten der BMAS-Erhebung zeigen, dass sich die Arbeitgeber teilweise aus der Finanzierung der bAV zurückziehen (Abbildung 5). Dies bedeutet nicht, dass Arbeitgeber insgesamt weniger für die bAV aufwenden, sondern kann damit zusammenhängen, dass sich deren Beiträge nun (gleichmäßiger) auf mehr Beschäftigte mit bAV-Anwartschaft verteilen bzw. neue Beschäftigte in die Mischfinanzierung einsteigen. Gegenüber 2001 haben

11 Hierbei handelt es sich um „Vollzeitbeschäftigte sowie entsprechend ihrer tatsächlich geleisteten Arbeitsstunden in Vollzeitarbeitsplätze umgerechnete Teilzeitbeschäftigte." (Statistisches Bundesamt 2018). 
sich die Arbeitgeber aus der alleinigen bAV-Finanzierung zurückgezogen, so dass diese Finanzierungsform im Jahr 2015 nur noch bei $28 \%$ aller privatwirtschaftlichen Unternehmen mit bAV anzutreffen war (gegenüber $54 \%$ im Jahr 2001). Dagegen war im Jahr 2015 die gemeinsame Finanzierung durch Arbeitgeber und ArbeitnehmerInnen die am häufigsten anzutreffenden Finanzierungsform der bAV $(60 \%$ aller Unternehmen mit bAV).

In Bezug auf die Unternehmensgröße kann festgestellt werden, dass die Beiträge zur bAV, vor allem durch die Arbeitgeber, sehr stark mit der Größe des Unternehmens ansteigen. Während Arbeitgeber in Unternehmen mit 10 bis 49 Beschäftigten 1,3\% der Bruttoverdienstsumme je Vollzeiteinheit für die bAV im Jahr 2016 aufgewendet haben, steigt dieser Wert kontinuierlich auf 5,1\% in Unternehmen mit 1000 Beschäftigten und mehr (siehe Abbildung 6).

Abbildung 6: Arbeitgeber- und Arbeitnehmeraufwendungen für die bAV 2016 je Vollzeiteinheit (\% Bruttoverdienstsumme) nach Unternehmensgröße

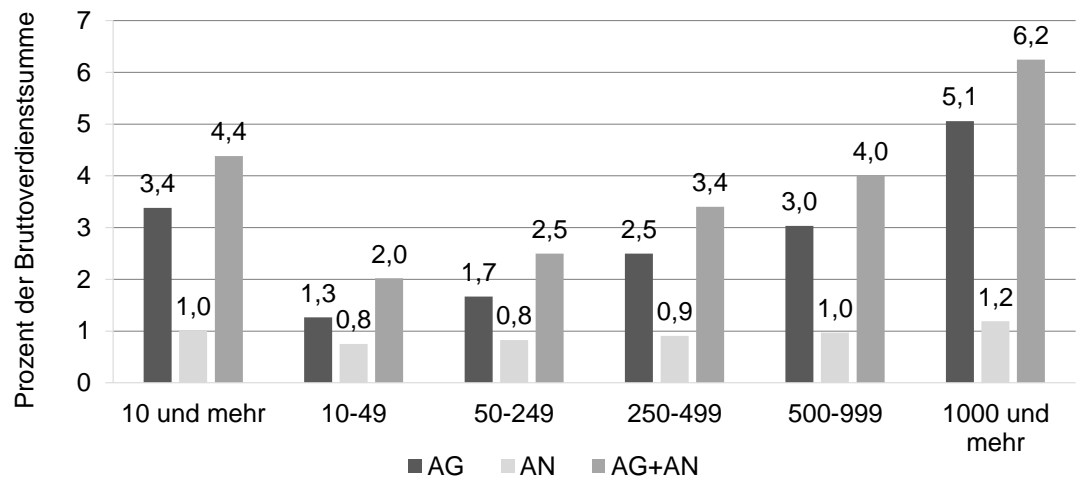

Quellen: Statistisches Bundesamt (2018).

Anmerkungen: siehe Fußnote 10.

\subsection{Branchenunterschiede}

Die Betrachtung nur des Landesdurchschnitts verdeckt erhebliche innerstaatliche Unterschiede, beispielsweise zwischen Branchen. Da die bAV oftmals Gegenstand von Verhandlungen zwischen Arbeitgebern oder de- 
ren Verbänden auf der einen Seite und Betriebsräten oder Gewerkschaften auf der anderen Seite ist, rücken Branchenunterschiede in den Fokus (für Details zu einzelnen Branchen, vgl. Wiß 2011). Unterschiede in der Struktur der Beschäftigten und deren Tätigkeiten, in der wirtschaftlichen Situation und der gesamtwirtschaftlichen Bedeutung, der Lohnhöhe, den Verteilungsspielräumen, und der Stärke von Gewerkschaften sowie der Tradition der Sozialpartnerschaft finden sich vor allem entlang von Branchen und bilden unterschiedliche Möglichkeiten und Bedingungen für die bAV (Blank 2015). Unterschiedliche Entwicklungen von bestimmten Teilsystemen unterhalb der nationalstaatlichen Ebene, wie zum Beispiel Branchen, können zudem potenziell wichtige Antreiber von institutionellem Wandel auf nationaler Ebene sein bzw. diesen signalisieren.

\section{Abbildung 7: Aufwendungen für die bAV 2016 je Vollzeiteinheit (\% Bruttover-} dienstsumme) nach Branche

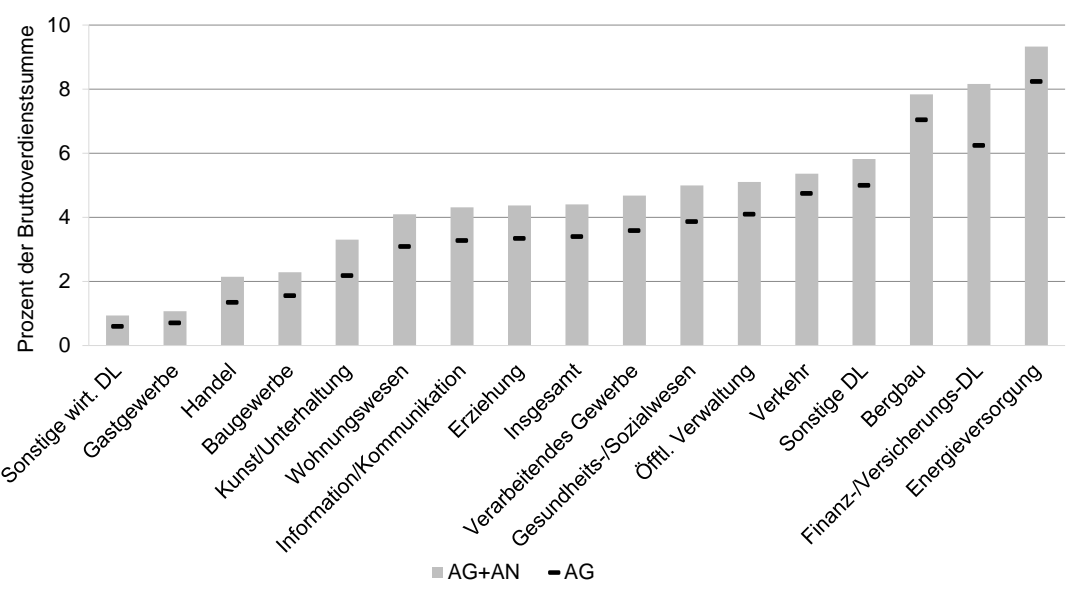

Quellen: Statistisches Bundesamt (2018).

Anmerkungen: siehe Fußnote 10. Die Werte für Energieversorgung beziehen sich auf das Jahr 2012.

Für die Betrachtung von Branchenunterschieden eignet sich insbesondere die Arbeitskostenerhebung des Statistischen Bundesamtes. Die aktuellste Erhebung für das Jahr 2016 zeigt, dass die bAV-Aufwendungen je Vollzeiteinheit bezogen auf die Bruttoverdienstsumme aller Beschäftigten in den sonstigen wirtschaftlichen Dienstleistungen $(0,9 \%)$, dem Hotel- und Gaststättengewerbe $(1,1 \%)$ sowie im Handel $(2,1 \%)$ am geringsten sind (siehe 
Abbildung 7). Dagegen liegen die Beiträge zur bAV vor allem in der Energieversorgung $(9,3 \%)$, den Finanz- und Versicherungsdienstleistungen $(8,2 \%)$ sowie im Bergbau (7,8\%) klar über dem Durchschnitt von 4,4\%. Leider beinhalten die Daten der Arbeitskostenerhebung des Jahres 2016 keine Informationen zur Verbreitung der bAV. Für genauere Analysen, auch zum Zusammenhang von Verbreitung und Beitragshöhe der bAV, müssen wir uns daher auf die Daten aus dem Jahr 2012 beschränken.

Im Gegensatz zur BMAS-Erhebung und zur Arbeitskostenerhebung 2016 beinhalten die Arbeitskostenerhebungen der Jahre 2012 und 2008 Informationen zur Anzahl der Beschäftigten mit bAV sowie den Aufwendungen hierfür von ArbeitnehmerInnen und Arbeitgebern, jeweils gegliedert nach Branchen. Auf dieser Basis kann nun entsprechend dem Anteil von Beschäftigten mit einer bAV die Bruttolohnsumme von Beschäftigten mit bAV ermittelt werden, unter der Annahme, dass alle Beschäftigten den gleichen Lohn erhalten. Die durchschnittlichen bAV-Aufwendungen durch Arbeitgeber und ArbeitnehmerInnen entsprechen ebenfalls dem Anteil der Beschäftigten mit bAV bzw. deren Bruttoverdienstsumme. Als Ergebnis erhalten wir eine Schätzung von bAV-Beiträgen pro ArbeitnehmerIn mit bAV. Dies ist daher nur eine grobe Annäherung auf Branchenebene, da keine genauen Informationen über einzelne Beschäftigte sowie Varianz und Verteilung in Bezug auf Löhne und Beiträge vorliegen. Selbst wenn somit die absoluten Werte nicht exakt der Realität entsprechen, erlaubt dieses Vorgehen die detaillierte Abbildung von Branchenunterschiede, die ansonsten nicht ermittelbar sind.

In Abbildung 8 sehen wir das Zusammenspiel von Arbeitgeberbeiträgen und Verbreitung der bAV. Im oberen rechten Quadranten befinden sich die Branchen, die sowohl bei der Höhe der Arbeitgeberbeiträge als auch bei der Anzahl der Beschäftigten mit bAV über dem Durchschnitt aller Branchen liegen. Die Branchen im unteren linken Quadranten dagegen weisen unterdurchschnittliche Werte bei beiden Dimensionen auf. Gegenüber vorherigen Berechnungen und den Abbildungen 6 und 7 muss bei der Höhe der Beiträge berücksichtigt werden, dass hier nun die bAV-Aufwendungen in Relation zur anteiligen Bruttoverdienstsumme der Beschäftigten mit bAV gesetzt werden und nicht zur Bruttoverdienstsumme aller Beschäftigten (Beschäftigte ohne bAV fließen also nicht in die Analyse ein).

Es besteht ein mittelstarker positiver Zusammenhang zwischen Höhe des Arbeitgeberbeitrages und der Verbreitung der bAV ( $\mathrm{r}=0,538)$. Dies bedeutet, dass die Anzahl der ArbeitnehmerInnen mit bAV mit der Höhe des Arbeitgeberbeitrages ansteigt. Demzufolge weisen vor allem die Finanzdienstleistungen und die Energieversorgung stark ausgebaute bAV-Syste- 
me auf. Im öffentlichen Dienst verfügen zudem dank eines Obligatoriums alle Beschäftigten über eine bAV und die Höhe der Arbeitgeberbeiträge entspricht dem Durchschnitt aller Branchen. Dagegen sind die bAV-Systeme im Gastgewerbe, im Handel und in den sonstigen wirtschaftlichen Dienstleistungen sehr lückenhaft und wenn vorhanden zahlen die Arbeitgeber nur geringe Beiträge. ${ }^{12}$

\section{Abbildung 8: ArbeitnehmerInnen mit bAV und Arbeitgeberbeiträge nach Bran- che 2012}

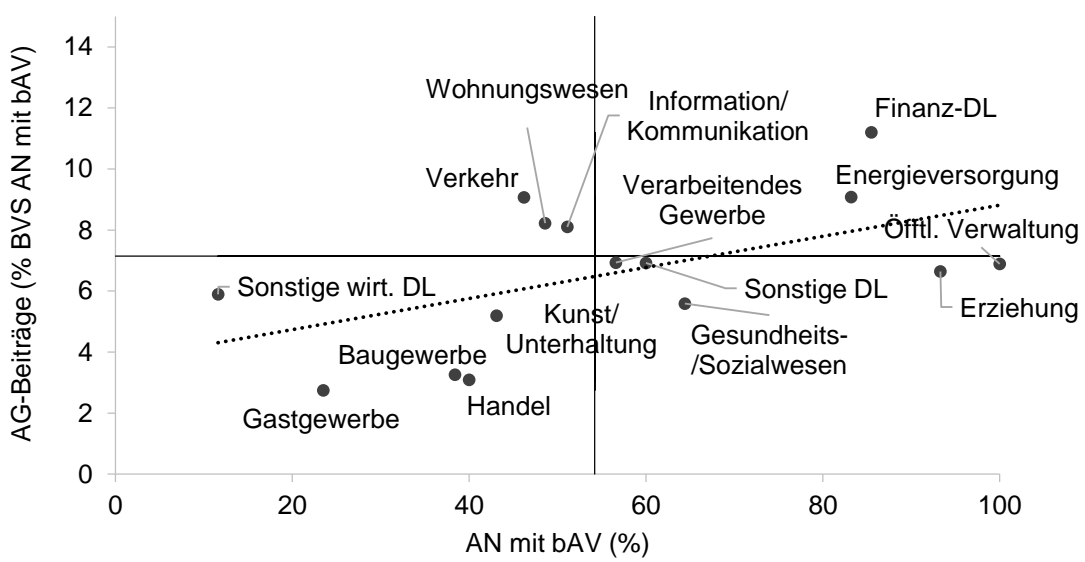

Quelle: Statistisches Bundesamt (2015b)

Anmerkungen: Siehe Fußnote 10. Die schwarzen Linien markieren den Durchschnitt aller Branchen.

Die bAV ist insgesamt in denjenigen Branchen recht weit verbreitet und mit zum Teil sehr generösen Arbeitgeberbeiträgen ausgestattet, in denen die Gewerkschaften stark sind und sich in Verhandlungen mit Arbeitgebern durchsetzen konnten (kollektive politische Machtressourcen) oder in

12 Die Verbreitung der bAV im Baugewerbe wird durch die Daten vermutlich unterschätzt, da es im Baugewerbe bis 2016 eine umlagefinanzierte Rentenbeihilfe gab, die seitdem schrittweise durch die kapitalfundierte Tarifrente Bau ersetzt wird. Bis zum Jahr 2016 war diese jedoch nur in Westdeutschland allgemeinverbindlich. Unklar ist, ob sich die Zahlen des Statistischen Bundesamtes lediglich auf die freiwillige BauRente ZukunftPlus beziehen. Unabhängig davon sind die Arbeitgeberbeiträge jedoch unter dem Durchschnitt aller Branchen. 
denen es überdurchschnittlich viele Beschäftigte mit hohen Qualifikationen gibt, so dass diese dank ihrer individuellen ökonomischen Machtressourcen über bAV-Anwartschaften verfügen (Wiß 2015).

Im Vergleich mit den Arbeitnehmerbeiträgen weisen Arbeitgeberbeiträge eine wesentliche höhere Varianz bzw. Standardabweichung bei insgesamt wesentlich höheren Beiträgen auf. Der Zusammenhang zwischen Höhe der Arbeitnehmerbeiträge und Verbreitung der bAV ist zudem negativ $(\mathrm{r}=-0,579)$ (siehe Abbildung 9). Dies bedeutet, dass der Anteil von Beschäftigten mit bAV mit der Höhe das Arbeitnehmerbeitrages sinkt, wobei hier eher nicht von einem kausalen Zusammenhang ausgegangen werden kann. Vielmehr liegt die Vermutung nahe, dass dies an geringen oder fehlenden Arbeitgeberbeiträgen liegt. In Branchen mit geringer bAV-Abdeckung wie den sonstigen wirtschaftlichen Dienstleistungen, Kunst und Unterhaltung sowie im Handel leisten die (wenigen) Beschäftigten mit bAV überdurchschnittlich hohe eigene Beiträge zur bAV. Dies sind vermutlich vor allem diejenigen Beschäftigten, die auch über höhere Löhne verfügen. Die Arbeitnehmerbeiträge zur bAV sind dagegen unterdurchschnittlich im öffentlichen Dienst und der Energieversorgung, wo aufgrund von hohen Arbeitgeberbeiträgen eine geringere Notwendigkeit von hohen eigenen Beiträgen besteht. Die Situation im Gastgewerbe ist besonders prekär, hier verfügen nur sehr wenige Beschäftigte über eine bAV und die Beiträge sowohl von Arbeitgebern als auch von ArbeitnehmerInnen sind unterdurchschnittlich.

\section{Abbildung 9: ArbeitnehmerInnen mit bAV und Arbeitnehmerbeiträge nach}

Branche 2012

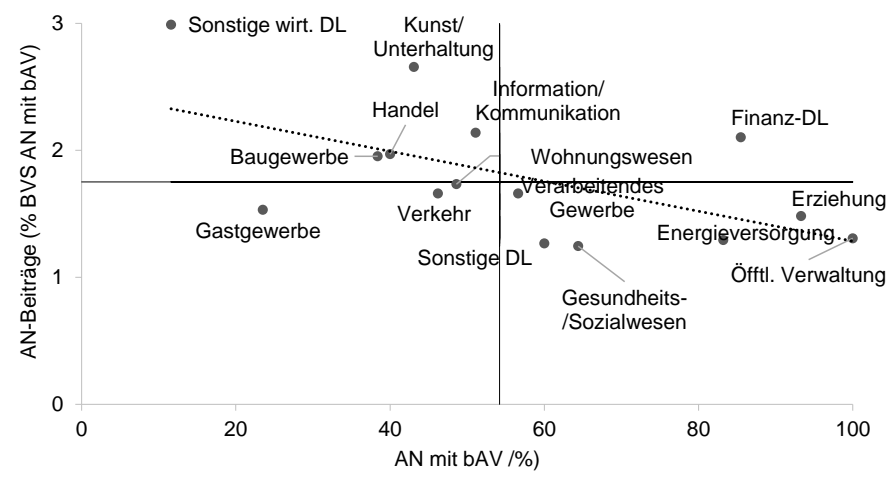

Quelle: Statistisches Bundesamt (2015b).

Anmerkungen: Siehe Abbildung 8. 
Damit lässt sich als Zwischenfazit festhalten, dass auf nationaler Ebene nur etwas mehr als die Hälfte aller Beschäftigten über eine bAV-Anwartschaft verfügen und die durchschnittliche Höhe der Beiträge kaum dazu beiträgt den Lebensstandard zusammen mit staatlichen Rentenleistungen zu sichern, wenn Direktzusagen und Unterstützungskassen, die sich eher an Beschäftigte mit höheren Einkommen richten, außen vorgelassen werden. Unterhalb der nationalen Ebene sind zudem signifikante Unterschiede zwischen einzelnen Branchen ersichtlich. Beide Ergebnisse sind darauf zurückzuführen, dass sowohl der Abschluss eines bAV-Vertrages und auch der Zuschuss von Arbeitgebern weitestgehend freiwillig erfolgt. Die politisch angedachte Kompensation eines Teiles der Lebensstandardsicherung durch die bAV Anfang der 2000er Jahre scheint damit in weiter Ferne und birgt zudem die Gefahr von Dualisierungsprozessen und verstärkten Ungleichheiten (Pavolini/Seeleib-Kaiser 2018). Der anschließende Abschnitt gibt daher einen kurzen Überblick, wie und vor allem wo, also in welchem System, andere europäische Länder die Lebensstandardsicherung im Alter organisieren.

\section{Erfahrungen anderer Länder als Reformoptionen?}

Die vorangegangen Analysen haben gezeigt, dass die Lebensstandardsicherung im Zusammenspiel aus staatlicher und betrieblicher Rente für die kommenden Generationen in Deutschland nicht (mehr) gewährleistet ist, was auf gekürzte staatliche Rentenleistungen und die lückenhafte Verbreitung der auf Freiwilligkeit basierenden bAV zurückzuführen ist. ${ }^{13}$ Wie aber sieht die Situation in anderen Ländern aus, was kann Deutschland hier möglicherweise von den anderen Ländern lernen? ${ }^{14}$

\subsection{Umlagefinanzierte Lebensstandardsicherung}

Eine erste Gruppe von Ländern hält nach wie vor an der Sicherung des Lebensstandards im Alter basierend alleinig auf der umlagefinanzierten staatlichen Rente fest. Dies trifft vor allem auf Italien und Österreich zu. Die aktuellen Zahlen der OECD, welche als eine von wenigen Quellen einen

13 Zur internationalen Außenseiter-Rolle Deutschlands vgl. Meyer 2015.

14 Für Details zu einzelnen Ländern vgl. Anderson 2017; Ebbinghaus 2011b; Immergut et al. 2007; Meyer et al. 2007; Natali 2017. 
internationalen Vergleich ermöglichen, weisen eine Nettoersatzrate von $51,9 \%$ für Deutschland, 91,8 \% für Österreich und 93,2 \% für Italien auf (OECD 2019). ${ }^{15}$ Selbst inklusive der freiwilligen bAV bzw. Riester-Vorsorge kommt Deutschland lediglich auf einen Wert von $68 \%$. Die Lebensstandardsicherung alleine durch das öffentliche System ist nur mit höheren Beiträgen finanzierbar, nämlich $22,8 \%$ in Österreich und $33 \%$ in Italien gegenüber 18,6\% in Deutschland (2018). In beiden Ländern zahlt der Arbeitgeber mehr als die ArbeitnehmerInnen. Ohne hier in Details gehen zu wollen ${ }^{16}$, gibt es also durchaus (noch) Länder, die an hohen staatlichen Rentenleistungen festhalten (in Abbildung 10 sind die grau markierten Bereiche für die Lebensstandardsicherung zuständig). Auch die Armutsvermeidung ist in diesen Ländern gegenüber Deutschland stärker in der ersten staatlichen Rentensäule verankert. Während Deutschland in der Grundsicherung im Alter ein Leistungsniveau in Höhe von $20 \%$ des Durchschnittslohns erreicht, kommt die Ausgleichszulage in Österreich auf einen Wert von $30 \%$ und die soziale Rentenzulage in Italien auf 21,1 \% (OECD 2019). ${ }^{17}$ Zusätzlich zur staatlichen Rentenversicherung verfügen Beschäftigte in Österreich und Italien, im Gegensatz zu Deutschland, über obligatorische Abschlagszahlungen, die für die bAV genutzt werden können. In Österreich zahlt der Arbeitgeber 1,53\% des Gehalts für jede/n Beschäftigte/ $\mathrm{n}$ in eine Mitarbeitervorsorgekasse ein (Abfertigung $\mathrm{Neu}$ ), welche die Beiträge an den Kapitalmärkten anlegt. Wenn die Beschäftigten ihr Unternehmen verlassen, kann das akkumulierte Kapital unter anderem in einen bAV-Vertrag eingezahlt werden. ${ }^{18}$ In ähnlicher Weise fungiert die Abschlagszahlung TFR in Italien als Einfallstor für die bAV, wobei der Arbeitgeberbeitrag mit 6,91\% wesentlich höher ist.

15 Abweichend von den OECD-Berechnungen vor 2019 wie in Abbildung 2 wird für den Bericht 2019 ein/e ArbeitnehmerIn, der mit 22 Jahren (und nicht wie vorher mit 20 Jahren) in das Erwerbsleben eintritt und entsprechend den Regeln im Jahr 2018 Rentenleistungen erwirbt als Berechnungsgrundlage genommen (OECD 2019).

16 Für den Vergleich von Deutschland mit Österreich vgl. Blank et al. 2016.

17 In Österreich gibt es zudem noch die bedarfsorientierte Mindestsicherung.

18 Die freiwillige bAV in Österreich dient dagegen weniger als Vorbild (vgl. Wiß 2018). 
Abbildung 10: Länder mit umlagefinanzierter Lebensstandardsicherung

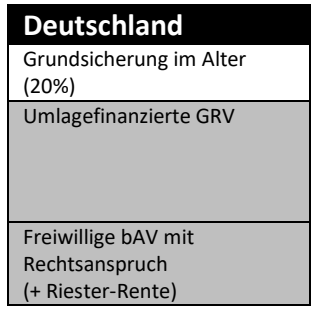

Quelle: eigene Darstellung
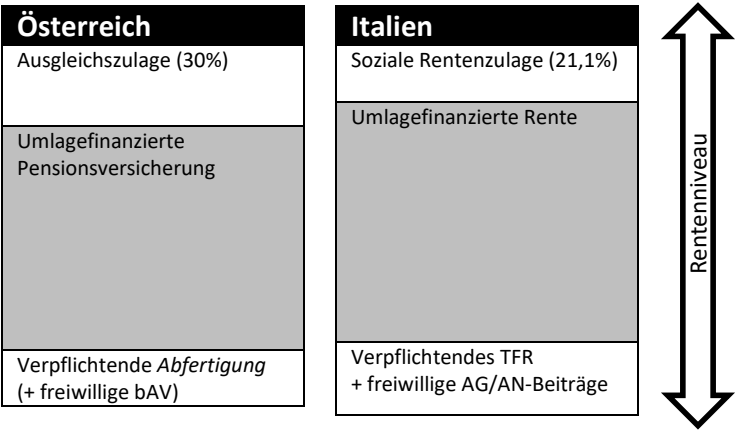

\subsection{Staatliche Armutsvermeidung und automatischer bAV-Einbezug}

Im Gegensatz zu Österreich und Italien besteht das vorrangige Ziel des staatlichen Alterssicherungssystems in Großbritannien in der Vermeidung von Altersarmut. Daher kommt der Basisrente in der ersten staatlichen Säule die größte Bedeutung zu. Jedoch ist das britische Leistungsniveau der Basisrente nach 35 Versicherungsjahren ${ }^{19}$ im Verhältnis zum Durchschnittslohn mit knapp $22 \%$ (OECD 2019) nur unwesentlich höher als die deutsche Leistung der Grundsicherung im Alter. Auch bei der Nettoersatzrate steht Großbritannien trotz jüngster Reformanstrengungen nicht besser da als Deutschland. Das staatliche System allein kann 28,4\% des vorherigen Einkommens substituieren, bei Berücksichtigung der bAV steigt dieser Wert auf $61 \%$ (gegenüber $68 \%$ in Deutschland inkl. freiwilliger privater Vorsorge; OECD 2019). Aufgrund der höheren Verpflichtung der bAV in Großbritannien (automatischer Einbezug mit Widerspruchsrecht) werden aber wohl wesentlich mehr Personen auch diese $61 \%$ erreichen, als Personen die in Deutschland auf $68 \%$ kommen. Die Beitragszahlungen zur britischen bAV setzen sich aus $3 \%$ Arbeitergeber-, $4 \%$ ArbeitnehmerIn- und $1 \%$ staatlichem Beitrag zusammen. Es ist fraglich, inwiefern Deutschland etwas vom britischen Alterssicherungssystem lernen kann. Der automatische Einbezug aller Beschäftigten in die bAV mit Wider-

19 In Großbritannien werden Zeiten ohne Erwerbstätigkeit sehr großzügig angerechnet, so zum Beispiel 11 Jahre pro Kind. 
spruchsrecht wäre sicherlich ein gangbarer Weg für mehr Verpflichtung auch in der deutschen bAV und ist auch bereits regelmäßig Bestandteil von Reformüberlegungen.

Abbildung 11: Staatliche Armutsvermeidung und automatischer bAV-Einbezug

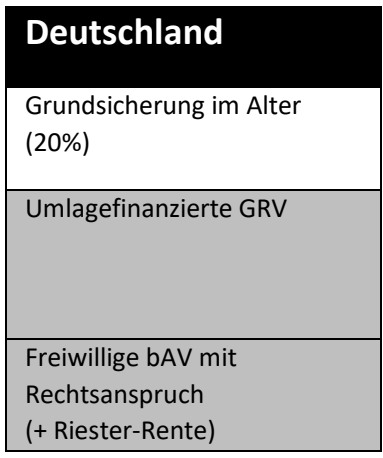

Quelle: eigene Darstellung
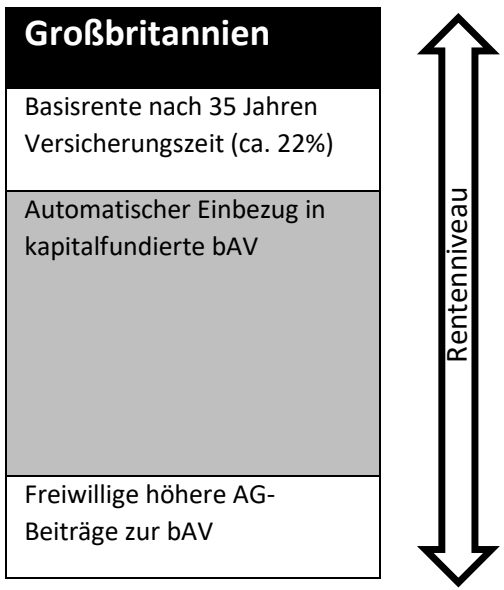

\subsection{Staatliche Armutsvermeidung und kapitalfundierte Lebensstandardsicherung}

Einen anderen Weg in Bezug auf Lebensstandardsicherung und bAV-Obligatorium sind die Niederlande, Dänemark und Schweden gegangen. ${ }^{20}$ Vor allem das niederländische und dänische System beinhalten Elemente, die für deutsche Reformdiskussion interessant sein könnten, da angesichts einer immer stärker werdenden Abhängigkeit künftiger Renteneinkommen von Finanzmärkten (Finanzialisierung) in Deutschland (Wiß 2019) höhere staatliche Renten in absehbarer Zeit politisch nicht umsetzbar erscheinen. ${ }^{21}$ Dank ihrer universalen Grundrentensysteme, deren einzige Voraussetzung der Wohnsitz im jeweiligen Land ist, leistet die staatliche

20 Für Details vgl. Anderson 2017.

21 Einen guten Überblick über den Forschungsstand und die Ausgestaltung der bAV aus internationaler Perspektive bieten Grødem et al. (2018) sowie Pavolini/ Seeleib-Kaiser (2018). 
erste Säule mit einem Leistungsniveau von $29 \%$ des Durchschnittslohns in den Niederlanden und sogar $37 \%$ in Dänemark einen starken Beitrag zur Eindämmung von Altersarmut (OECD 2019). ${ }^{22}$ Die schwedische Mindestrente, die in Zukunft auslaufen wird, bietet ähnlich der deutschen Grundsicherung im Alter ein Niveau von 22,3\%.

\section{Abbildung 12: Länder mit kapitalfundierter Lebensstandardsicherung}
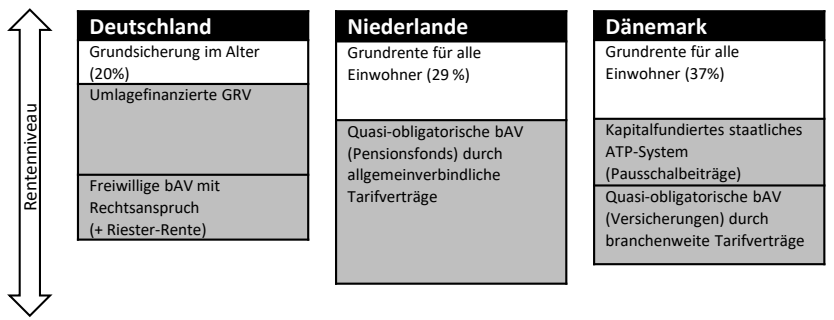

\begin{tabular}{|l|}
\hline Schweden \\
\hline Garantierente $(21,4 \%)$ \\
\hline $\begin{array}{l}\text { Umlagefinanzierte } \\
\text { einkommensbezogene Rente }\end{array}$ \\
\hline $\begin{array}{l}\text { Verpflichtende individuelle } \\
\text { kapitalfundierte Rente }\end{array}$ \\
\hline $\begin{array}{l}\text { Quasi-obligatorische bAV } \\
\text { (Versicherungen) durch } \\
\text { Tarifverträge }\end{array}$ \\
\hline
\end{tabular}

Quelle: eigene Darstellung

Beim Blick auf die Nettoersatzraten wird deutlich, dass Schweden, oftmals von politischen Akteuren in Deutschland als Vorbild propagiert, mit $53,4 \%$ Deutschland mit 51,9\% nur unwesentlich übertrifft. Wesentlich besser stehen dagegen die Niederlande (80,2 \%) und Dänemark (70,9\%) da (OECD 2019), wobei die OECD gegenüber 2017 nun zu wesentlich geringeren Werten kommt. ${ }^{23}$ Die zentrale Rolle der bAV in diesen Ländern für die Lebensstandardsicherung wird deutlich, wenn man die Nettoersatzraten nur für die bAV berechnet. Diese beträgt $40 \%$ in den Niederlanden (also in etwa so viel wie die staatliche Säule), $50 \%$ in Dänemark und ca. $10-15 \%$ in Schweden (OECD 2019). Kennzeichen aller drei Länder ist die sozialpartnerschaftliche Organisation der bAV (Anderson 2019). In Schweden tragen zusätzlich die umlagefinanzierte staatliche Rente und die verpflichtende individuelle kapitalfundierte Prämiepension zum Lebensstandard im Alter bei. In allen drei Ländern wenden die Arbeitgeber wesentlich höhere Beiträge auf als die ArbeitnehmerInnen. In den Niederlanden bewegt sich der Gesamtbeitragssatz zur bAV zwischen $15 \%$ und $25 \%$ und

22 Hier muss berücksichtigt werden, dass diese Zahlen keine Ersatzraten sind, sondern ähnlich dem deutschen Sicherungsniveau die Rentenhöhe ins Verhältnis zum Durchschnittslohn der jeweils aktuellen Beschäftigten setzt.

232017 errechnete die OECD eine Nettoersatzrate für die Niederlande in Höhe von 100,6 \% und für Dänemark 80,2 \% (OECD 2017). 
in Dänemark zwischen $10 \%$ bis $17 \%$, wobei in beiden Ländern die Arbeitgeber ca. zwei Drittel und die ArbeitnehmerInnen ein Drittel übernehmen (Anderson 2017). ${ }^{24}$ In Schweden wird die bAV dagegen alleine durch den Arbeitgeber finanziert (4,5\%). Insgesamt zeigt sich, dass alle Länder, die eine wesentlich höhere Rentenersatzrate als Deutschland aufweisen, hierfür auch mehr finanzielle Beiträge aufwenden (vor allem die Arbeitgeber), unabhängig davon ob die Lebensstandardsicherung über Steuern und Umlagefinanzierung oder über die Kapitalfundierung erfolgt.

\section{Fazit}

Ziel dieses Kapitels war es, die Rolle der bAV im deutschen Alterssicherungssystem zu analysieren, vor allem im Hinblick auf deren Entwicklung seit der Rentenreform von 2001. In Relation zu allen sozialversicherungspflichtig Beschäftigten konnte die bAV Anfang der 2000er Jahre starke Zugewinne verbuchen, seit 2005 allerdings stagniert die bAV und ist in den letzten Jahren sogar rückläufig. Dies hat enorme negative Folgen für die Lebensstandardsicherung der heutigen und künftigen Beschäftigten, da die Rentenreformen der letzten 20 Jahre das Leistungsniveau der gesetzlichen Rentenversicherung gesenkt haben. Dies ist das Ergebnis der Fehleinschätzung deutscher Politik, dass eine auf Freiwilligkeit basierende bAV die entstandene Lücke für den Lebensstandard füllen kann. Die Freiwilligkeit der bAV hat zugleich neuen Ungleichheiten Vorschub geleistet, z.B. zwischen Männern und Frauen, West- und Ostdeutschland und entlang von Branchen. Aktuell kann das deutsche Alterssicherungssystem, entgegen dem ursprünglichen Ziel, keine Lebensstandardsicherung im Alter mehr gewährleisten. Ein Blick auf andere Länder zeigt, dass wesentlich mehr finanzielle Mittel hierfür aufgewendet werden müssten. Dabei erscheinen zwei Wege als zielführend. Entweder geht man den Weg von Österreich und Italien (und den früheren Weg von Deutschland) und stärkt massiv die gesetzliche Rentenversicherung um den Lebensstandard über den staatlichen Teil des Alterssicherungssystems zu sichern. Oder man orientiert sich konsequent an den Niederlanden und Dänemark, wo die steuerfinanzierte Basisrente für alle Einwohner Altersarmut verringert und die Lebensstandardsicherung in der quasi-obligatorischen bAV, basierend auf Tarifverträgen

24 Der aktuelle OECD Bericht weist folgende Beitragssätze zur bAV aus: NL: 14,8 \% Arbeitgeber (AG), 7,7\% ArbeitnehmerIn (AN); DK: $8 \%$ AG, $4 \%$ AN; SE: AG: $4,5 \%$ (OECD 2019). 
und gemeinsam verwalteten Versorgungsystemen, erfolgt. Beide Wege bringen ihre jeweils eigenen Nachteile mit sich, zum Beispiel bezüglich ungünstiger demografischer Entwicklungen, finanzieller Nachhaltigkeit und unvorhersehbaren Finanzmarktentwicklungen.

Die deutsche Politik hat versucht, mit dem Betriebsrentenstärkungsgesetz des Jahres 2017 auf die fehlende Dynamik in der bAV zu reagieren. Um die bAV in Deutschland zu stärken, setzte die Große Koalition auf eine stärkere Verbreitung über Tarifverträge. Das seit Januar 2018 mögliche Sozialpartnermodell, bei dem Gewerkschaften und Arbeitgeber gemeinsam betriebliche Versorgungsysteme einrichten und verwalten können, kommt jedoch nicht in Gang. Auch die nun rechtssichere Option, per Tarifvertrag den automatischen Einbezug von Beschäftigten in die bAV mit Widerspruchsrecht zu regeln, konnte sich bisher nicht durchsetzen. Lediglich ein Unternehmen plant demnächst einen Haustarifvertrag unter dem Dach des Sozialpartnermodells abzuschließen. Das Versicherungsunternehmen Talanx hat in Kooperation mit der Gewerkschaft ver.di das Sozialpartnermodell „Die deutsche Betriebsrente“ vereinbart, das den Beschäftigten voraussichtlich im Jahr 2020 zur Verfügung stehen wird (Talanx 2019).

Mitverantwortlich für das zurückhaltende Engagement der Gewerkschaften ist sicherlich auch die im Sozialpartnermodell vorgesehene reine Beitragszusage, die im Gegensatz zu bisherigen bAV-Verträgen keine Leistungsgarantie mehr vorsieht. Im Falle negativer Finanzmarktentwicklungen wären die Gewerkschaften letztendlich mitverantwortlich für geringer als geplante Rentenleistungen und womöglich Kürzungen in der Auszahlungsphase. Eine weitere Hürde für das Sozialpartnermodell stellt die Tarifbindung dar. Diese ist in den letzten Jahren kontinuierlich zurückgegangen, so dass aktuell fast jeder zweite Beschäftigte in einem Betrieb ohne Tarifbindung arbeitet.

Hier kommt der fehlende Mut der deutschen Alterssicherungspolitik zum Vorschein. Erneut wird davor zurückgescheut, mehr Verbindlichkeit in die bAV einzubauen. Stattdessen setzt man den bisher wenig erfolgreichen Weg der freiwilligen bAV fort, in der (eher fehlgeleiteten) Hoffnung, die Anzahl von Beschäftigten mit bAV über finanzielle Anreize und Förderungen unter Einbezug der Sozialpartner zu erhöhen. Die bislang fehlende Verpflichtung in der bAV bewahrt aber zugleich auch die Chance, die Lebensstandardsicherung künftiger Generationen doch wieder stärker über die staatliche Rente zu gewähren. Hierfür wäre allerdings ein breiter politscher Wille, dafür auch höhere Beiträge zu erheben, notwendig. Höhere staatliche Renten scheinen aber in Deutschland in absehbarer Zeit poli- 
tisch eher nicht durchsetzbar zu sein. Die Leidtragenden dieser fehlenden Klarheit deutscher Rentenpolitik sind künftige Rentnerinnen und Rentner.

\section{Literatur}

Anderson, K.M. (2017): Anpassung der Alterssicherungssysteme an das veränderte Marktumfeld - ein internationaler Vergleich am Beispiel der Staaten Schweden, Niederlande und Dänemark, in: Deutsche Rentenversicherung 2017 (4), S. 440456

Anderson, K.M. (2019): Financialisation meets collectivisation: occupational pensions in Denmark, the Netherlands and Sweden, in: Journal of European Public Policy 26 (4), S. 617-636

Blank, F. (2015): Die betriebliche Altersversorgung als Gegenstand der Sozialpolitik - Stand der Dinge und Weiterentwicklung, in: Sozialer Fortschritt 64 (8), S. $184-189$

Blank, F./Logeay, C./Türk, E./Wöss, J./Zwiener, R. (2016): Österreichs Alterssicherung: Vorbild für Deutschland? In: Wirtschaftdienst 96 (4), S. 279-286

Bridgen, P./Meyer, T. (2014): The liberalisation of the German social model: public-private pension reform in Germany since 2001, in: Journal of Social Policy 43 (1), S. 37-68

Bundesagentur für Arbeit (2019): Beschäftigte nach ausgewählten Merkmalen (Zeitreihe Quartalszahlen), Nürnberg

Bundesministerium für Arbeit und Soziales (BMAS; 2008): Ergänzender Bericht der Bundesregierung zum Rentenversicherungsbericht 2008 (Alterssicherungsbericht 2008), Berlin

Bundesministerium für Arbeit und Soziales (2016): Forschungsbericht 475-Arbeitgeber- und Trägerbefragung zur Verbreitung der betrieblichen Altersversorgung 2015, Berlin

Bundesministerium für Arbeit und Soziales (2019a): Bericht der Bundesregierung -Rentenversicherungsbericht 2019, Berlin

Bundesministerium für Arbeit und Soziales (2019b): Forschungsbericht 523-Trägerbefragung zur Verbreitung der betrieblichen Altersversorgung 2017, Berlin

Bundesministerium für Arbeit und Soziales (2019c): Statistik zur privaten Altersvorsorge (Riester-Rente), https:/www.bmas.de/DE/Themen/Rente/ZusaetzlicheAltersvorsorge/statistik-zusaetzliche-altersvorsorge.html (abgerufen am 05.12.2019).

Bundesministerium für Arbeit und Soziales (2020): Überblick über die steuer- und sozialabgabenrechtliche Behandlung von Aufwendungen für die betriebliche Altersvorsorge, https:/www.bmas.de/SharedDocs/Downloads/DE/ueberblick-ue ber-die-steuer-und-sozialversicherungsrechtliche-behandlung-von-aufwendunge $\mathrm{n}$-fuer-die-betriebliche-altersvorsorge.pdf?_blob=publicationFile\&v $=3$ (abgerufen am 10.02.2020) 
Deutsche Rentenversicherung Bund (2018): Rentenversicherung in Zeitreihen 2018 - DRV Schriften Band 22, Berlin

Ebbinghaus, B. (2011): Introduction: studying pension privatization in Europe, in: Ebbinghaus, B. (Hrsg.): The Varieties of Pension Governance - Pension Privatization in Europe, Oxford, S. 3-22

Ebbinghaus, B. (2018): Privatisierung und Vermarktlichung der Altersvorsorge: Eingetrübte Aussichten des deutschen Mehrsäulenmodells, in: WSI Mitteilungen 71 (6), S. 468-475

Ebbinghaus, B./Gronwald, M./Wiß, T. (2011): Germany: departing from Bismarckian public pensions, in: Ebbinghaus, B. (Hrsg.): The Varieties of Pension Governance - Pension Privatization in Europe, Oxford, S. 119-150

Grødem, A.S./Hagelund, A./Hippe, J.M./Trampusch, C. (2018); Beyond coverage: the politics of occupational pensions and the role of trade unions - Introduction to special issue, in: Transfer: European Review of Labour and Research, 24 (1), S. $9-23$

Immergut, E.M./Anderson, K.M. (2007): Editors' introduction: the dynamics of pension politics, in: Immergut, E.M./Anderson, K.M./Schulze, I. (Hrsg.): The Handbook of West European Pension Politics, Oxford, S. 1-45

Meyer, T. (2015): Die dritte Säule in der Alterssicherung - brauchen wir eine Neubewertung nach der Finanz- und Wirtschaftskrise? In: Sozialer Fortschritt 64 (8), S. $189-195$

Nullmeier, F. (2015): Einstürzende Neubauten - Statikprobleme im Säulenmodell der Alterssicherung, in: Sozialer Fortschritt 64 (8), S. 196-202.

OECD (2005): Pensions at a Glance 2005, Paris

OECD (2009): Pensions at a Glance 2009, Paris

OECD (2017): Pensions at a glance 2017, Paris

OECD (2019): Pensions at a glance 2019, Paris

Pavolini, E./Seeleib-Kaiser, M. (2018): Comparing occupational welfare in Europe: the case of occupational pensions, in: Social Policy \& Administration 52 (2), S. $477-490$

Schulze, I./Jochem, S. (2007): Germany: beyond policy gridlock, in: Immergut, E.M./Anderson, K.M./ Schulze, I. (Hrsg.): The Handbook of West European Pension Politics, Oxford, S. 660-710

Statistisches Bundesamt (2011): Verdienste und Arbeitskosten - Aufwendungen und Anwartschaften betrieblicher Altersversorgung 2008, Wiesbaden

Statistisches Bundesamt (2015a): bAV in der AKE 2012-Forschungsvorhaben zur Sicherung und Verbesserung der Qualität statistischer Daten über den Verbreitungsgrad betrieblicher Altersversorgung aus der Erhebung der Struktur der Arbeitskosten, Wiesbaden

Statistisches Bundesamt (2015b): Verdienste und Arbeitskosten - Aufwendungen und Anwartschaften betrieblicher Altersversorgung 2012, Wiesbaden 
Statistisches Bundesamt (2018): Verdienste und Arbeitskosten 2016, Bonn

Talanx (2019): Die Deutsche Betriebsrente kommt: Ver.di wird Sozialpartner; https://www.talanx.com/newsroom/unternehmensmeldungen/2019/2019-10-17. aspx?sc_lang=de-de (abgerufen am 06.12.2019)

Wiß, T. (2011): Der Wandel der Alterssicherung in Deutschland Die Rolle der Sozialpartner, Wiesbaden

Wiß, T. (2015): From welfare states to welfare sectors: explaining sectoral differences in occupational pensions with economic and political power of employees, in: Journal of European Social Policy 25 (5), S. 489-504

Wiß, T. (2018): Divergent occupational pensions in Bismarckian countries: the case of Germany and Austria, in: Transfer: European Review of Labour and Research 24 (1), S. 91-107

Wiß, T. (2019): Reinforcement of pension financialisation as a response to financial crises in Germany, the Netherlands and the United Kingdom, in: Journal of European Public Policy 26 (4), S. 501-520 
